\title{
ON CHARACTERS IN THE PRINCIPAL 2-BLOCK, II
}

\author{
MARCEL HERZOG and CHERYL E. PRAEGER
}

(Received 30 August 1977; revised 18 December 1978)

Communicated by M. H. Newman

\begin{abstract}
Let $k$ be a non-zero complex number and let $u$ and $v$ be elements of a finite group $G$. Suppose that at most one of $u$ and $v$ belongs to $O(G)$, the maximal normal subgroup of $G$ of odd order. It is shown that $G$ satisfies $X(v)-X(u)=k$ for every complex nonprincipal irreducible character $X$ in the principal 2-block of $G$, if and only if $G / O(G)$ is isomorphic to one of the following groups: $C_{2}, P S L\left(2,2^{n}\right)$ or $P \Sigma L\left(2,5^{2 a+1}\right)$, where $n \geqslant 2$ and $a \geqslant 1$.
\end{abstract}

Subject classification (Amer. Math. Soc. (MOS) 1970): 20 C 20

\section{Introduction}

Let $G$ be a finite group. It was shown by Berger and Herzog (1978) that if $u \in G$ and $k \in \mathbf{C}$ satisfy:

$$
X(1)-X(u)=k
$$

for every complex non-principal irreducible character in the principal 2-block of $G$, then either $u \in O(G)$ or $G / O(G)$ is isomorphic to one of the following simple groups: $C_{2}, \operatorname{PSL}\left(2,2^{n}\right), n \geqslant 2$. The converse also holds.

The aim of this paper is to consider the more general equality

$$
X(v)-X(u)=k,
$$

where $k$ is a non-zero complex number, $v, u \in G$ and (1) holds for every complex non-principal irreducible character in the principal 2-block of $G$. In this case we obtain new candidates for $G / O(G)$, namely $P \Sigma L\left(2,5^{2 a+1}\right), a \geqslant 1$, the extension of $P S L\left(2,5^{2 a+1}\right)$ by the group of automorphisms of the Galois field with $5^{2 a+1}$ elements.

The first author was supported by a grant from the Israel Commission for Basic Research. 
Our main result is

THEOREM 1. Let $G$ be a finite group, $u$ and $v$ be elements of $G$ and $k$ be a non-zero complex number. Suppose that (1) is satisfied by every complex non-principal irreducible character of $G$ belonging to $B$, the principal 2-block of $G$. Then, either $\{u, v\} \cap O(G) \mid=1$ or $k= \pm 4$ and $G / O(G)$ is isomorphic to $P \Sigma L\left(2,5^{2 a+1}\right), a \geqslant 1$.

We also prove the following

Proposition. Let $G=P \Sigma L\left(2,5^{2 a+1}\right), u \in G$ be an involution and $v \in G$ be of order $2 a+1$ such that $G=\left\langle P S L\left(2,5^{2 a+1}\right), v\right\rangle$. Then (1) holds for every complex nonprincipal irreducible character in the principal 2-block of $G$, with $k=4$.

The authors are grateful to the referee for providing the proof of the Proposition.

Combining these results with the Theorem of Berger and Herzog (1978), we get

THEOREM 2. $G$ satisfies the assumptions of Theorem 1 , if and only if $G / O(G)$ is isomorphic to one of the following groups: $C_{2}, P S L\left(2,2^{n}\right), n \geqslant 2$ and $P \Sigma L\left(2,5^{2 a+1}\right)$, $a \geqslant 1$.

In this paper $G$ denotes a finite group. The order of $G$ is $g$ and if $v \in G, o(v)$ denotes the order of $v$. The principal 2-block of $G$ is denoted by $B$, and the number of irreducible characters in $B$ is $b$. The letter $X$ will always denote an irreducible character in $B(X \in B)$. A fixed Sylow 2-subgroup of $G$ will be denoted by $S$. If $H$ is a subgroup of $G$ and $v \in G$, then $o(v \bmod H)$ is the least positive integer $n$ satisfying $v^{n} \in H$, and $\exp H$ is the least positive integer $m$ satisfying: $h^{m}=1$ for every $h \in H$. The group of outer automorphisms of $H$ will be denoted by Out $H$. We denote by $\Sigma$ or $\Sigma^{\#}$ the summation over all $X \in B$ or $X \in B \backslash 1_{G}$, respectively. The expression 'the orthogonality relations in blocks' will be abbreviated by O.R.B. Finally, $C_{2}$ will denote the cyclic group of order 2 .

\section{Proof of Theorem 1}

It is well known that $O(G)=\bigcap\{\operatorname{ker} X \mid X \in B\}$. As $k \neq 0$, it follows that not both $u$ and $v$ belong to $O(G)$. So assume that $u, v \notin O(G)$ and it suffices to prove the theorem under the assumption that $O(G)=1$.

It is well known that if $y \in G$, then $\Sigma^{\sharp} X(y)$ is a rational integer. Thus, by (1), $(b-1) k \in Z$ and since $X(v)-X(u)$ is an algebraic integer, we conclude that

$$
k \in Z-\{0\} .
$$


Suppose that $y \in G$ does not belong to the 2-sections of either $v$ or $u$ in $G$. Then, by (1) and the O.R.B.,

$$
0=\Sigma X(y)(X(v)-X(u))=k \Sigma^{\sharp} X(y)
$$

yielding

$$
\Sigma^{\sharp} X(y)=0 .
$$

It follows that $y \neq 1$ and consequently we may assume without loss of generality that

$$
v \text { has odd order, } o(v)>1 \text {. }
$$

Let $w$ be a 2-element of $G$ of maximal order, and let $z$ be the involution in $\langle w\rangle$. Then, by the O.R.B., $\Sigma X(1) X(z)=0$, and since as in Berger and Herzog (1978)

$$
X(w) \equiv X(z) \equiv X(1) \quad(\bmod \mathscr{P}),
$$

where $\mathscr{P}$ is the prime ideal lying over 2 in $\mathcal{O}$, the integers in $Q(\sqrt[g]{ } 1)$, it follows that

Hence

$$
\Sigma^{\sharp} X(1) \equiv \Sigma^{\sharp} X^{2}(1) \equiv \Sigma^{\#} X(1) X(z) \equiv 1 \quad(\bmod 2) .
$$

$$
\Sigma^{\sharp} X(w) \equiv \Sigma^{\sharp} X(z) \equiv \Sigma^{\sharp} X(1) \equiv 1 \quad(\bmod 2) .
$$

Thus, in view of (3) and (4), w=z and we get

(7) $\exp S=2$, where $S$ is a Sylow 2-subgroup of $G$,

(8) $G$ has one class of involutions, and

(9) $o(u)=2 f$, where $f$ is an odd integer.

In particular, $G$ has exactly two 2 -sections.

Choose $H$, a minimal normal subgroup in $G$. As $O(G)=1$, it follows by (8) that $G / H$ is of odd order and as in Berger and Herzog (1978), either $H=S$ or $H$ is isomorphic to one of the following simple groups: $\operatorname{PSL}(2, q), q>3, q \equiv 0,3$ or 5 ( $\bmod 8), J$ (Janko's smallest group) or $\operatorname{Re}(q)$ (a group of Ree type). Since none of the above-mentioned groups satisfies (1) for a $v$ satisfying (4), it follows that

(10) $G / H$ is a non-trivial soluble group of odd order.

Let $Y$ be a non-principal linear character of $G / H$ and suppose that $Y \in B$. Clearly, by (1) and (2), $k= \pm 1$ or \pm 2 . If $k= \pm 2$, then by (1) $\{Y(v), Y(u)\}=\{1,-1\}$, which is impossible since $G / H$ is of odd order. If $k= \pm 1$, then by (1)

$$
\{Y(v), Y(u)\}=\left\{\exp \left(\frac{1}{3} \pi i\right), \exp \left(\frac{2}{3} \pi i\right)\right\} \text { or }\left\{\exp \left(\frac{4}{3} \pi i\right), \exp \left(\frac{5}{3} \pi i\right)\right\},
$$


again in contradiction to (10). Thus:

(11) No non-principal linear character of $G / H$ belongs to $B$.

Proceeding exactly as in Berger and Herzog (1978), we get

(12) $G=C_{d}(S) H$,

(13) $H$ is non-abelian simple,

(14) $G / H$ is isomorphic to a subgroup of Out $H$,

(15) $H \neq J, P S L\left(2,2^{n}\right), n \geqslant 2$, and

(16) If $Y$ is an irreducible character of $G / H$ belonging to $B$, then $Y=1$.

Suppose that $H \simeq \operatorname{Re}(q)$. As in Berger and Herzog (1978), $B$ consists of 8 characters $X_{i}, i=1, \ldots, 8$, such that $\left.X_{i}\right|_{H}=\xi_{i}, i=1, \ldots, 8$. We use here the notation of Ward (1966) for the irreducible characters and elements of $H$. By the O.R.B., (1), (4) and (9) we get

$$
0=\Sigma X(v) X(u)=1+k \Sigma^{\sharp} X(u)+\Sigma^{\sharp}|X(u)|^{2}
$$

whence

$$
0=k \Sigma^{\sharp} X(u)+\Sigma|X(u)|^{2} .
$$

In addition, the O.R.B. yield:

$$
0=\sum X(u) X(R)=X_{1}(u)+X_{2}(u)+X_{3}(u)+X_{4}(u)
$$

and

$$
0=\Sigma X(u)(3 X(R)+X(S)+X(V)+X(W))=6 X_{1}(u)+6 X_{2}(u) .
$$

As $X_{1}(u)=1,(18)$ and (19) yield:

$$
X_{2}(u)=-X_{1}(u)=-1, \quad X_{4}(u)=-X_{3}(u) .
$$

The O.R.B. also yield:

$$
0=\Sigma X(u) X(Y)=m\left(X_{5}(u)+X_{6}(u)+X_{7}(u)+X_{8}(u)\right)
$$

whence

$$
X_{5}(u)+X_{6}(u)+X_{7}(u)+X_{8}(u)=0 .
$$

It follows from (17), (18) and (21) that

$$
k=\Sigma|X(u)|^{2} .
$$


Applying the O.R.B. to $v$ we get

$$
0=\sum X(v) X(J R)=X_{1}(v)-X_{2}(v)+X_{3}(v)-X_{4}(v),
$$

which implies in view of (1) and (20)

$$
X_{3}(u)=-X_{4}(u)=(k-2) / 2 .
$$

Thus $k$ is even, and by (20), (22) and (23):

$$
k \geqslant 1+1+(k-2)^{2} / 2 \text {. }
$$

It follows that one of the following holds:

$$
k=4, \quad X_{i}(u)=0 \quad \text { for } i=5,6,7,8,
$$

or

$$
k=2, \quad X_{i}(u)=0 \quad \text { for } i=3,4,5,6,7,8 .
$$

Another application of the O.R.B. yields, in view of (1), (20) and (23),

$$
0=\Sigma X(v) X(J S)=1-(k-1)-(3 k / 2-1)+(k / 2+1)
$$

so that $k=2$.

A final application of the O.R.B., together with (20), yields:

$$
0=\sum X(u) X(1)=1+(-1)\left(q^{2}-q+1\right)=q(1-q),
$$

a contradiction.

Finally, suppose that $H \cong P S L(2, q), q>5$ and $q \equiv 3$ or $5(\bmod 8)$. As in Berger and Herzog (1978), $B$ consists of 4 characters $X_{i}, i=1, \ldots, 4$, such that $\left.X_{i}\right|_{H}=\theta_{i}$, $i=1, \ldots, 4$. We use here the notation of Ward (1966), pp. 62-65, for the irreducible characters and elements of $\boldsymbol{H}$. By the O.R.B. we have

$$
0=\Sigma X(u) X(R)=X_{1}(u)-e X_{4}(u),
$$

where $e= \pm 1$ satisfying $q \equiv 4+e(\bmod 8)$, as defined in Ward's paper. Hence,

$$
X_{4}(u)=e \text {. }
$$

Thus, again by the O.R.B.,

yielding

$$
0=\Sigma X(u) X(1)=1+(q+e)\left(X_{2}(u)+X_{3}(u)\right) / 2+e q
$$

$$
X_{2}(u)+X_{3}(u)=-2 e .
$$

A final application of the O.R.B., together with (1), (24) and (25), yields

$$
0=\Sigma X(v) X\left(S_{0}^{(q-e) / 4}\right)=1-2 k e+2+e k+1,
$$

whence $k=4 e$ and $X_{4}(v)=5 e$. 
Now by (10) and (14)

$$
P S L(2, q) \subset G \subseteq P \Sigma L(2, q) .
$$

Thus $G$ has a 2-transitive permutation representation of degree $q+1$, the restriction of which to $H$ is also 2-transitive. Let $Y$ be the irreducible character of $G$ of degree $q$ corresponding to this representation. Then $\left.Y\right|_{H}$ is irreducible, and since $Y(1)=q$, $\left.Y\right|_{H}=\theta_{4}=\left.X_{4}\right|_{H}$ and consequently $X_{4}=Y \cdot \xi$, where $\xi$ is a linear character of the cyclic group $G / H$ (see Isaacs (1976), (6.17)). Thus $5 e=X_{4}(v)=Y(v) \xi(v)$, where $Y(v)$ is an integer $\geqslant-1$ and $\xi(v)$ is an odd root of 1 . We conclude that $e=1$ and $Y(v)=5$. So $v$ fixes exactly $5+1=6$ elements in the permutation representation of $G$. Let $q=p^{c}$ and let $o(v \bmod H)$ be $d$. Then $d$ divides $c$ and

$$
6=\operatorname{fix}(v)=1+p^{c / d} \text {. }
$$

Consequently $p=5$ and $d=c$; as $5^{c}=q \equiv 4+e=5(\bmod 8), c=2 a+1$ for some $a \geqslant 1$. Since $o(v \bmod H)=c=2 a+1$, by (26) $G=P \sum L\left(2,5^{2 a+1}\right)$, and the proof of Theorem 1 is complete.

\section{Proof of the Proposition}

Let $2 a+1=r, q=5^{r}$ and let $H \triangleleft G, H \simeq P S L(2, q)$. Since $|G: H|=r$, then $u \in H$. It follows from the arguments of Section 2 that the principal 2-block $B$ of $G$ consists of 4 irreducible characters: $X_{i}, i=1, \ldots, 4$, such that $\left.X_{i}\right|_{H}=\theta_{i}$, $i=1, \ldots, 4$. For the irreducible characters and elements of $H$ we use again the notation of Ward (1966), pp. 62-65.

As in Section 2, $G$ has an irreducible character $Y$ of degree $q$ corresponding to the 2-transitive permutation representation of $G$ of degree $1+q$ on $\Omega$, and again $\left.Y\right|_{H}$ is irreducible, whence $\left.Y\right|_{H}=\theta_{4}$. Being the unique extension of $\theta_{4}$ which is rational, $Y \in B$ forcing $Y=X_{4}$. Moreover, $Y(u)=1$ and $Y(v)=5$ since $v$ fixes exactly 6 elements of $\Omega$. Thus $X_{4}$ satisfies (1) with $k=4$, and it suffices to show that also $X_{2}$ and $X_{3}$ do so. By the O.R.B. we have:

$$
0=\Sigma X(u) X(v)=1-X_{2}(v)-X_{3}(v)+5
$$

whence $X_{2}(v)+X_{3}(v)=6$. As $X_{2}(u)=X_{3}(u)=-1$, it suffices to show that $X_{2}(v)=3$. In particular, it suffices to show that $\psi=\theta_{2}$ has an extension $\psi$ to $G$ with $\psi(v)=3$, since being the unique extension of $\psi$ which is rational on $v, \hat{\psi} \in B$ whence $\psi=X_{2}$.

Let $R=\langle v\rangle$ and choose $Q \in \operatorname{Syl}_{5}(H)$ and a cyclic subgroup $C$ of $H$ of order $(q-1) / 2$, such that $N \equiv N_{H}(Q)=Q C$ and $R \subseteq N_{G}(Q) \cap N_{G}(C)$. It follows from the character table of $H$ that $\left.\psi\right|_{N}=\theta+\lambda$, where $\theta$ is irreducible of degree $(q-1) / 2$ and $\lambda^{2}=1_{N}$. Now $\theta$ has a unique extension $\hat{\theta}$ to $N R$ such that $\hat{\theta}$ is real (see Isaacs 
(1970), Theorems 11.22 and 6.17, remembering that $N$ has 2 irreducible characters of degree $(q-1) / 2$ and $|N R: N|$ is odd). It can be shown similarly, that $\psi$ has a unique extension $\psi$ to $G$ such that $\left.\hat{\psi}\right|_{N R}$ contains $\hat{\theta}$ as a component. Thus $\left.\hat{\psi}\right|_{N R}=\hat{\theta}+\hat{\lambda}$, where $\hat{\lambda}$ is an extension of $\lambda$. Since $\hat{\psi}$ is unique and $\hat{\theta}$ is real, so also $\hat{\psi}$ is real, forcing $\hat{\lambda}(v)$ to be real. Consequently $\hat{\lambda}(v)=1$ and it suffices to show that $\hat{\theta}(v)=2$.

Since $\theta$ is a character of $N$ induced from $Q,\left.\theta\right|_{C}$ is the regular character of $C$. Write the representation which affords $\left.\hat{\theta}\right|_{C R}$ with reference to a basis consisting of eigenvectors for a generator $c$ of $C$. As the eigenvectors correspond to distinct eigenvalues, and as $v$ normalizes $C$, the matrix representing $v$ must be monomial, and has precisely two zero entries on the diagonal (namely, in the positions corresponding to the eigenvalues +1 and -1 of $c$; no other $\frac{1}{2}(q-1)$ th root of 1 is invariant under the fifth powering action of $v$ ). Thus $\left.\hat{\theta}\right|_{C R}=\mu+\nu+\tau$, where $\mu$ and $\nu$ are linear characters and $\tau$ is a character vanishing on $v$ and on $u v$, where $u$ denotes an involution in $C$. Since $\hat{\theta}$ is real, $\mu+\nu$ is real on $v$ and on $u v$. Choose the notation so that $\mu(u)=1=-v(u)$. Then both $(\mu+v)(v)=\mu(v)+\nu(v)$ and $(\mu+\nu)(u v)=\mu(v)-v(v)$ are real, forcing $\mu(v)$ and $\nu(v)$ to be real. Consequently $\mu(v)=v(v)=1$ and $\hat{\theta}(v)=2$, as required.

\section{References}

Thomas R. Berger and Marcel Herzog (1978), 'On characters in the principal 2-block', J. Austral. Math. Soc., (Ser. A) 25, 264-268.

I. Martin Isaacs (1976), Character theory of finite groups (Academic Press, New York, San Francisco and London).

Harold N. Ward (1966), 'On Ree's series of simple groups', Trans. Amer. Math. Soc. 121, 62-89.

Department of Mathematics

Institute of Advanced Studies

The Australian National University

Canberra, Australia 2600

and

Department of Mathematics

Tel-Aviv University

Tel-Aviv, Israel
Department of Mathematics

University of Western Australia

Nedlands, Australia 6009 